expiration, but not necessarily the groaning. Although most patients show normal laryngoscopic findings during daytime examination, some authors suggest a partial closure of the glottis as the cause of the groaning, but this has never been proven.

We performed laryngoscopy during deep sedation using propofol in a 29-yr-old female without any comorbidites who was transferred for the evaluation of nocturnal groaning, EDS (Epworth Sleepiness Scale 17 out of 24 points), and exerciseinduced and occasional nocturnal dyspnoea, starting 15 yrs previously. A pulmonological examination, including bronchoscopy, revealed neither asthma nor any stenosis of the upper airways or the trachea.

The otorhinolaryngological examination showed a decreased mobility of the right vocal cord; there was a slight medial dislocation of the right arytenoid, but a cervical computed tomography scan revealed neither lysis nor dislocation or malformation of the arytenoids. Phonation was not affected.

A video polysomnogaphy showed an apnoea-hypopnoea index of 5 events $\cdot h^{-1}$; minimal oxygen saturation was $94 \%$. Snoring was not detectable but during major periods in rapid eye movement (REM) and non-REM sleep, groaning and prolonged expiration were observed (fig. 1a-b); diagnostic criteria for catathrenia were fulfilled and all major differential diagnoses were excluded.

Laryngoscopy under deep sedation showed the open glottis at inspiration, but during expiration, a subtotal closure of the glottis was seen, causing the groan that is typical for catathrenia (fig. 1c and d). During the groaning, the vocal cords perform an expiratory active adduction followed by a vibration; thus, the mechanism of the groaning is similar to a normal phonation.

Due to the EDS, we started treatment with nasal CPAP at a fixed pressure of $8 \mathrm{cmH}_{2} \mathrm{O}$, leading to a significant reduction in nocturnal groaning and improved wellbeing during the daytime.
This is the first description of a laryngoscopy in a sleeping patient with catathrenia. The active adduction of the vocal cords during expiration strongly supports the hypothesis of a phonation causing the groaning and, thus, of the parasomnic nature of catathrenia. As long as many unanswered questions remain, especially addressing the underlying pathophysiological mechanism of expiratory active closure of the vocal cords, attempting a CPAP trial in patients who may want treatment will be reasonable.

\section{S.R. Ott*, J. Hamacher* and E. Seifert ${ }^{\#}$}

*Dept of Respiratory and Sleep Medicine, and "Dept of Othorhinolaryngology, Inselspital, University Hospital and University of Berne, Berne, Switzerland.

Correspondence: S.R. Ott, Dept of Respiratory and Sleep Medicine, Inselspital, University Hospital Berne, CH-3010 Berne, Switzerland. E-mail: Sebastian.ott@insel.ch

Statement of Interest: None declared.

\section{REFERENCES}

1 De Roek J, van Hoof E, Cluydts R. Sleep-related expiratory groaning. A case report. J Sleep Res 1983; 12: 237.

2 American Academy of Sleep Medicine. International Classification of Sleep Disorders, 2nd Edn: Diagnostic and Coding Manual. Westchester, American Academy of Sleep Medicine, 2005.

3 Guilleminault C, Hagen CC, Khaia AM. Catathrenia: parasomnia or uncommon feature of sleep disordered breathing? Sleep 2008; 31: 132-139.

4 Ortega-Albas JJ, Diaz JR, Serrano AL, et al. Continous positive airway pressure as a treatment for catathrenia (nocturnal groaning). Neurology 2006; 67: 1103.

\title{
The search for autoantibodies against elastin, collagen and decorin in COPD
}

\section{To the Editors:}

Several studies indicate that an autoimmune response may underlie the pathogenesis of chronic obstructive pulmonary disease (COPD). A high prevalence of anti-Hep-2 epithelial cell autoantibodies has been shown in patients with COPD [1, 2], as well as autoantibodies against airway epithelial cells [1], endothelial cells [3], lung elastin [4], several immunogenic peptides [5] and cytokeratin 18 [6]. The most convincing evidence for an autoimmune response in COPD was provided by LeE et al. [4]. They showed a specific autoantibody and a specific T-cell response against elastin in COPD patients, as well as increased numbers of anti-elastin antibody-producing cells in the lungs of COPD patients. Recent studies [7, 8], however, could not confirm such an anti-elastin autoantibody response. COTTIN et al. [7] found no evidence for an anti-elastin autoantibody response in patients with combined pulmonary fibrosis and emphysema. Additionally, GREENE et al. [8] investigated the presence of autoantibodies against elastin- and collagen-derived proline-glycine-proline in patients with COPD and $\alpha_{1}$-antitrypsin deficiency, and also did not observe increased autoantibody levels.

We assessed the presence of autoantibodies against elastin, collagen and decorin in serum of COPD patients and healthy controls. In addition, and as an extension of previous studies, we included equal numbers of current, ex- and never-smokers, in order to investigate the effect of the smoking status on 
autoantibody levels. We chose to asses autoantibodies against elastin and collagen to confirm the previous results of LEE et al. [4] in two different patient groups. As we previously demonstrated a decreased presence of the extracellular matrix protein decorin around airways of severe COPD patients [9], we additionally investigated autoantibodies against decorin.

Autoantibodies against elastin, collagen and decorin were measured using a slightly modified ELISA protocol, as described by LEE et al. [4] (online supplementary material provides detailed description of the methods), in two groups of COPD patients and healthy controls. The first group consisted of 19 COPD patients and 32 healthy controls derived from the Dutch part of the large population-based European Community Respiratory Health Study (ECRHS) [10]. The second group was newly recruited, and consisted of 23 COPD patients and 36 healthy controls. Participant characteristics of both groups are presented in table 1 . Both groups consisted of only males to exclude possible sex effects. Although COPD patients were slightly older than healthy controls, and COPD smokers and exsmokers had more pack-yrs of exposure than healthy smokers and ex-smokers, these differences did not affect serum autoantibody levels as assessed by linear regression analysis.

Initially, we assessed autoantibody levels in the ECRHS group and found that immunoglobulin (Ig)G autoantibody levels against elastin, collagen and decorin were not significantly different between COPD patients and healthy controls (fig. 1a, $c$ and e). The only significant difference observed was a higher level of anti-decorin IgG autoantibodies in COPD ex-smokers than COPD smokers. A similar, but nonsignificant, trend was found for autoantibodies against elastin and collagen.

To replicate our initial overall negative findings we decided to assess autoantibody levels in a second group of newly recruited
COPD patients and healthy controls. Again, we found no differences in autoantibody levels against elastin, collagen and decorin between COPD patients and healthy controls (fig. 1b, d and $\mathrm{f}$ ), thereby confirming our initial findings.

In the present study, we did not find an increased presence of autoantibodies against elastin, collagen and decorin in COPD, when comparing two separate groups of COPD patients and healthy control subjects. However, we did find an increased antidecorin autoantibody response in COPD ex-smokers compared with COPD smokers in one of the two groups.

Together with the results described by GREENE et al. [8], these data make a total of three different groups in which no increased anti-elastin autoantibody response was found in COPD. There may be several explanations for the discrepant findings compared with LEE et al. [4]. First, differences in the ethnicity and sex distributions of the populations, and also severity of COPD and presence of emphysema could have had an effect. We included only males to avoid effects of sex, while LEE et al. [4] included both males and females, with highest percentages of females in the healthy controls. With respect to the severity of COPD, we included relatively mild COPD patients, the majority having stage II disease; whereas, COPD severity in the study of LEE et al. [4] ranged from stage II to stage IV. Furthermore, LEE et al. [4] included COPD patients with established emphysema based on computerised tomography $(\mathrm{CT})$ quantification. These CT data were not available in our COPD patients. Based on these differences and considering the large range of autoantibody levels in COPD patients and control subjects, one could hypothesise that antielastin autoantibodies are more likely to be present in a subset of COPD patients having more severe disease and/or in patients with established emphysema.

TABLE 1 Participant characteristics

\begin{tabular}{|c|c|c|c|c|}
\hline & Subjects n & Age yrs & $\begin{array}{c}\text { Smoking exposure } \\
\text { pack-yrs }\end{array}$ & $\mathrm{FEV}_{1}{ }^{\#} \%$ pred \\
\hline \multicolumn{5}{|l|}{ ECRHS group } \\
\hline \multicolumn{5}{|l|}{ COPD } \\
\hline Smokers & 9 & $61(54-58)^{\star}$ & $33(26-63)^{*}$ & $75(42-87)$ \\
\hline Ex-smokers & 10 & $67(59-69)^{*}$ & $28(11-83)^{*}$ & $74(49-86)$ \\
\hline Ex-smokers & 10 & $55(47-70)$ & $19(11-29)$ & $114(97-138)$ \\
\hline Never-smokers & 12 & $55(47-68)$ & 0 & $123(103-154)$ \\
\hline \multicolumn{5}{|l|}{ Second group } \\
\hline \multicolumn{5}{|l|}{ COPD } \\
\hline Smokers & 12 & $67(45-76)^{\star}$ & $39(28-75)^{*}$ & $65(49-79)^{\bullet}$ \\
\hline Never-smokers & 12 & $58(52-74)$ & 0 & $110(94-129)$ \\
\hline
\end{tabular}

Data are presented as median (range), unless otherwise stated. FEV1: forced expiratory volume in $1 \mathrm{~s}$; \% pred: \% predicted; ECRHS: European Community Respiratory Health Study; COPD: chronic obstructive pulmonary disease. ${ }^{\#}$ : pre-bronchodilator FEV1, unless otherwise stated. ": post-bronchodilator FEV1. *: p<0.05 for COPD patients versus healthy controls. 

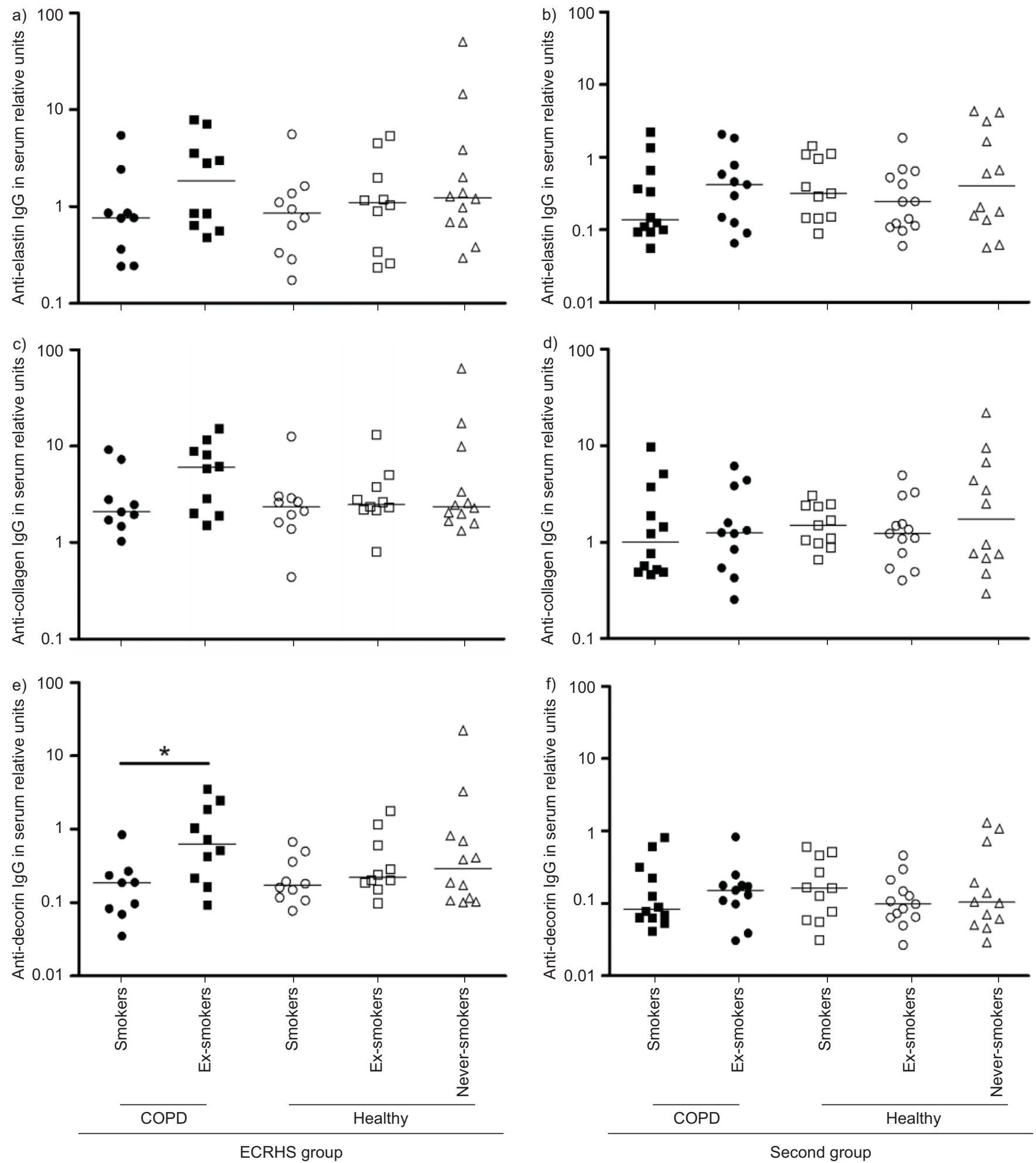

FIGURE 1. Serum levels of immunoglobulin (Ig)G antibodies against a, b) elastin, c, d) collagen and e, f) decorin in chronic obstructive pulmonary disease (COPD) patients (filled symbols) and healthy controls (open symbols) in a, c, e) the European Community Respiratory Health Study (ECRHS) group and b, d, f) the second group. -: median. *: $p<0.05$.

Given the positive correlation between the degree of emphysema and the specific T-cell response against elastin as shown by LEE et al. [4], it seems likely that this relationship with severity of emphysema also exists for autoantibody levels against elastin. This analysis was not reported by LEE et al. [4]. COTTIN et al. [7] and GREENE et al. [8], who both included COPD patients with established emphysema, could not detect this relationship with disease severity. 
Secondly, smoking history and current smoking status may also have an effect on autoantibody levels. In our study, higher autoantibody levels were present in COPD ex-smokers than COPD smokers. This is consistent with the study of LEE et al. [4], in which the majority of COPD patients were ex-smokers, and stresses the importance of including balanced numbers of current and ex-smokers to be able to analyse these groups separately.

Finally, it is likely that the autoantibody response in COPD can be directed against many different antigens, which may vary between individuals. The reported presence of autoantibodies against several antigens and cell types, such as airway epithelial cells, endothelial cells, lung elastin, immunogenic peptides and cytokeratin 18, is consistent with this reasoning. Such variability will make it more difficult to detect the presence of autoantibodies against a specific antigen in any given group. A more straightforward method to investigate the autoantibody response in COPD would then be to focus on the presence of specific antibody responses directed against lung tissue components, as FEGHALI-BOSTWICK et al. [1] did, when showing the presence of autoantibodies against lung epithelial cells and the deposition of IgG complexes in the lung.

In conclusion, we did not observe an increased autoantibody response against elastin in two separate groups of COPD patients. This is in agreement with two other recent studies, but discrepant from the first report of LEE et al. [4]. This discrepancy may indicate that the specific autoantibody response against elastin is not present in all COPD patients, but only in a specific subset. Furthermore, it may reflect that the autoantibody response in COPD can be directed against several different antigens, perhaps also varying from patient to patient, and thus, difficult to confirm in different patient groups. Additionally, we found differences in the autoantibody levels in COPD patients depending on the smoking status, which stresses the importance of taking this into account.

More studies are needed focusing on the presence of autoantibody responses directed against lung tissue components in COPD patients, particularly in relation to disease severity, smoking status and disease phenotype, i.e. the presence of emphysema.

\section{C.A. Brandsma*, H.A.M. Kerstjens", M. Geerlings*,} M. Kerkhof ${ }^{\varpi}$, M.N. Hylkema*, D.S. Postma" and W. Timens* *Dept of Pathology, "Dept of Pulmonary Diseases, and "Dept of Epidemiology, University Medical Center Groningen, University of Groningen, Groningen, The Netherlands.
Correspondence: C.A. Brandsma, Dept of Pathology and Medical Biology, University Medical Center Groningen, PO box 30.001, 9700 RB, Groningen, The Netherlands. E-mail: c.a.brandsma@path.umcg.nl

Support Statement: This study was funded by the Dutch Asthma Foundation (grant number 3.2.06.075).

Statement of Interest: None declared.

\section{REFERENCES}

1 Feghali-Bostwick CA, Gadgil AS, Otterbein LE, et al. Autoantibodies in patients with chronic obstructive pulmonary disease. Am J Respir Crit Care Med 2008; 177: 156-163.

2 Bonarius HP, Brandsma CA, Kerstjens HA, et al. Antinuclear autoantibodies are more prevalent in COPD in association with low body mass index but not with smoking history. Thorax 2011; 66: 101-107.

3 Karayama M, Inui N, Suda T, et al. Anti-endothelial cell antibodies in patients with COPD. Chest 2010; 138: 1303-1308.

4 Lee SH, Goswami S, Grudo A, et al. Antielastin autoimmunity in tobacco smoking-induced emphysema. Nat Med 2007; 13: $567-569$.

5 Leidinger P, Keller A, Heisel S, et al. Novel autoantigens immunogenic in COPD patients. Respir Res 2009; 10: 20.

6 Kuo YB, Chang CA, Wu YK, et al. Identification and clinical association of anti-cytokeratin 18 autoantibody in COPD. Immunol Lett 2010; 16, 128: 131-136.

7 Cottin V, Fabien N, Khouatra C, et al. Anti-elastin autoantibodies are not present in combined pulmonary fibrosis and emphysema. Eur Respir J 2009; 33: 219-221.

8 Greene CM, Low TB, O'Neill SJ, et al. Anti-proline-glycineproline or antielastin autoantibodies are not evident in chronic inflammatory lung disease. Am J Respir Crit Care Med 2010; 181: 31-35.

9 van Straaten JF, Coers W, Noordhoek JA, et al. Proteoglycan changes in the extracellular matrix of lung tissue from patients with pulmonary emphysema. Mod Pathol 1999; 12: 697-705.

10 Kerkhof M, Droste JH, de Monchy JG, et al. Distribution of total serum $\operatorname{IgE}$ and specific $\operatorname{IgE}$ to common aeroallergens by sex and age, and their relationship to each other in a random sample of the Dutch general population aged 20-70 years. Dutch ECRHS Group, European Community Respiratory Health Study. Allergy 1996; 51: 770-776. 